\title{
Origins of Extragalactic Cosmic Ray Nuclei by Contracting Alignment Pat- terns induced in the Galactic Magnetic Field
}

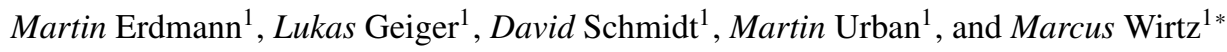 \\ ${ }^{1}$ III. Physikalisches Institut A, RWTH Aachen University
}

\begin{abstract}
We present a novel approach to search for origins of ultra-high energy cosmic rays. In a simultaneous fit to all observed cosmic rays we use the galactic magnetic field as a mass spectrometer and adapt the nuclear charges such that their extragalactic arrival directions are concentrated in as few directions as possible. During the fit the nuclear charges are constraint by the individual energy and shower depth measurements. We show in a simulated astrophysical scenario that source directions can be reconstructed even within a substantial isotropic background.
\end{abstract}

\section{Introduction}

Finding the sources of the ultra-high energy cosmic rays (UHECRs) is one of the fundamental questions in astroparticle physics and up to now still an unresolved puzzle. Recent studies revealed first a significant large scale anisotropy in form of a dipolar pattern [1] and then an indication for anisotropy on intermediate angular scales in form of a correlation with starburst galaxies [2] at the $4 \sigma$ confidence level.

Although there may be promising source candidates, a proof for a point source which is emitting UHECRs is still missing. A possibility to detect a point source would be the detection of a significant cosmic ray deflection pattern induced in cosmic magnetic fields. The deflections in the galactic magnetic field are likely to dominate over those from extragalactic fields. During the last years the knowledge about the galactic magnetic field is constantly increasing and there are models which were fitted to numerous rotation measurements and synchrotron radiation data [3-5]. These models contain a large scale field component that leads to a coherent deflection of UHECRs, proportional to the inverse of the magnetic rigidity $R=E / Z$ with the energy $E$ and the nuclear charge $Z$ of the UHECR candidate. Thus, it is expected that a closeby outstanding point source will cause an energy-ordering spectrometer effect in the arrival directions, which was examined in [6], however, the most significant found multiplet in data of the Pierre Auger Observatory had a chance probability of $\sim 5 \%$ to arise in isotropic distributions.

This work aims at finding these multiplets by including the charges of the UHECR into the alignment pattern, we call this mixed composition multiplets. Additionally we will make use of the preferred deflection directions by the magnetic field in our Galaxy. To visualize the challenge of finding a mixed composition multiplet, we first show in

*e-mail: mwirtz@physik.rwth-aachen.de

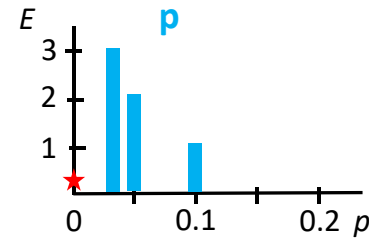

a)

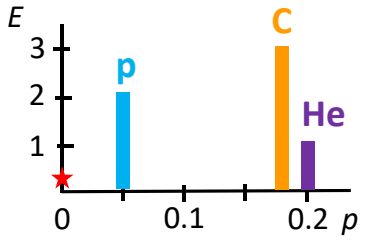

b)
Figure 1. One dimensional sketch of a) an only energy dependent multiplet structure of protons with $1 / E$ deflection behavior and of b) a mixed composition multiplet structure where the $1 / E$ deflection order is not visible anymore due to the proton, helium and carbon components. The red star at $p=0$ indicates the common source direction.

Fig. 1a an one dimensional sketch of the arrival directions $p$ of proton cosmic rays of different energies $E=1,2,3$ in arbitrary units, which all originate from the same source at $p=0$ (red star). There is visible a deflection pattern following $p \propto 1 / E$.

However, when we allow for a mixed composition and therefore for different charges of the cosmic ray candidates, this pattern will be disorganized up as sketched in Fig. 1b. Here the highest energy cosmic ray is a carbon nucleus with charge $Z=6$ and therefore 6 times more deflected than in Fig. 1a. Obviously, a method which only considers the cosmic ray energies without accounting for nuclear charges will not find the pattern anymore.

\section{Basic strategy}

The basic idea of the presented method is to start with a set of source positions and nuclear charges of the cosmic rays and project these assumptions to the observed arrival directions on Earth. This projection is done by assuming a 
a)

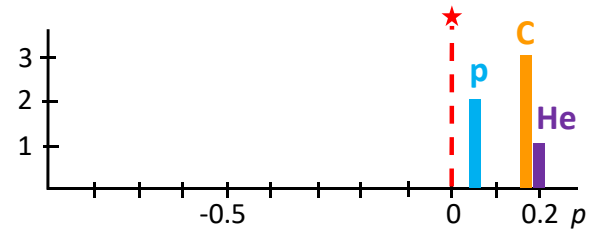

b) Prob.

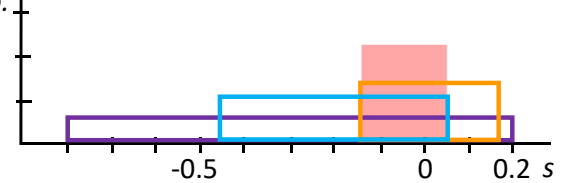

c)

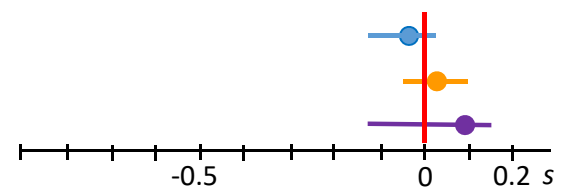

Figure 2. Reconstruction ansatz of a common source from three cosmic rays for the mixed composition case, a) true source with proton, helium and carbon nuclei, b) overlap window from maximum possible charges and corresponding deflections, c) weighted average source position from individual cosmic ray charge estimates.

galactic magnetic field model, in this work the model from Jansson \& Farrar [4] (further called JF12 model).

In Fig. 2 the transformation strategy is sketched by a simple toy transformation, where cosmic rays arrive in Fig. 2a with the measured arrival direction $p=s+Z / E$. In this example the source is located at $s=0$, the toycharges $Z$ are given by $1 / 26$ for the proton, $2 / 26$ for the helium and $6 / 26$ for the carbon nuclei, and the energy $E$ is denoted on the y-axis. Having only the information that the nuclear charges are between proton and iron, thus $Z=1, \ldots, 26$, leads to the picture in Fig. 2b. The assumption that all cosmic rays share a common origin constrains the source direction to the red shaded box. When having additional information via charge sensitive observables, like the shower depth maximum or the shape of time traces in surface detectors, we can constrain the source position further, as indicated in Fig. 2c. This toy example seems to be far from reality, as we expect strong isotropic contamination between the cosmic rays, which share a common origin. Note, however, that we are searching for nearly 1 dimensional structures caused by coherent magnetic fields on a 2-dimensional surface of a sphere, thus diminishing isotropic contamination on the direct line of deflection.

In the fit we will define a set of objective functions to constrain the measurements of the cosmic rays on Earth, namely arrival directions and shower depth maximum to stay within their experimental uncertainties. In the same step, the common initial source directions are clustered by varying the nuclear charges. After the fit we compare the cluster strengths to the ones found in isotropic arrival scenarios, defining us a statistical measure to discriminate signal enriched scenarios from isotropic ones.

\section{Techniques of the fit}

For each of the measured cosmic rays $i$, we have an observed arrival direction $p_{i}$, the energy $E_{i}$ and a charge sensitive observable, e.g. the shower depth maximum $X_{\max , \mathrm{i}}$.

We use a transformation prescription $T$ to predict for each given pair of fit parameters $\left(\hat{s}_{i}, \hat{Z}_{i}\right)$ of the estimated source direction $\hat{s}_{i}$ and the estimated nuclear charge $\hat{Z}_{i}$, the estimated arrival direction $\hat{p}_{i}$ on Earth:

$$
\left(\begin{array}{c}
\hat{s}_{i} \\
\hat{Z}_{i}
\end{array}\right) \Longrightarrow T\left(\hat{s}_{i}, \hat{Z}_{i}, E_{i}\right) \Longrightarrow \hat{p}_{i}
$$

In our notation all variables with a hat refer to variables directly related to the fit.

\subsection{Deflections in the galactic magnetic field}

The transformation $T$ in equation (1) represents the effect of the galactic magnetic field on the propagation of particles, where we use the model of Jansson \& Farrar [4] as mentioned above. For the fitting technique, our deflection model needs to be differentiable, thus, we trained a deep neural network consisting of 5 hidden layers with each 100 fully connected nodes to learn the projection from the inputs $\left(\hat{s}_{i}, R_{i}\right)$ to the output $\hat{p}_{i}$, where $R_{i}=\hat{Z}_{i} / E_{i}$ denotes the magnetic rigidity of the particle. We will refer to this neural network as MagNet. In Fig. 3 we compare the arrival directions of the simulation in the analytical JF12 field obtained from the software framework CRPropa3 [7] with the predictions of the MagNet for various rigidities $R_{i}=\hat{Z}_{i} / E_{i}$ and for two arbitrary chosen directions $\hat{s}_{i}$ outside our Galaxy. We find that the new differentiable model is well able to interpolate between rigidites and directions outside the Galaxy that the network has not "seen" during the training process.

\subsection{Objective function}

The objective function guides the fit parameters of equation (1) and consists of several terms which aim at concentrating cosmic ray directions prior to transformation while preserving the observed quantities, namely the arrival directions on Earth and the shower maximum.

Spatial distances between predicted and observed directions. As the initial source directions $\hat{s}_{i}$ are completely free fit parameters, the predicted arrival directions $\hat{p}_{i}$ have to be constrained to match the measured ones. This is done by introducing an objective term which penalizes the deviation between both variables:

$$
D=\frac{1}{N} \sum_{i}\left\|p_{i}-\hat{p}_{i}\right\|^{2}
$$

Differences between predicted and observed charges. The exact formulation of this objective term will depend on the given charge sensitive observable. In our benchmark fit we will assume that we have the shower depth 


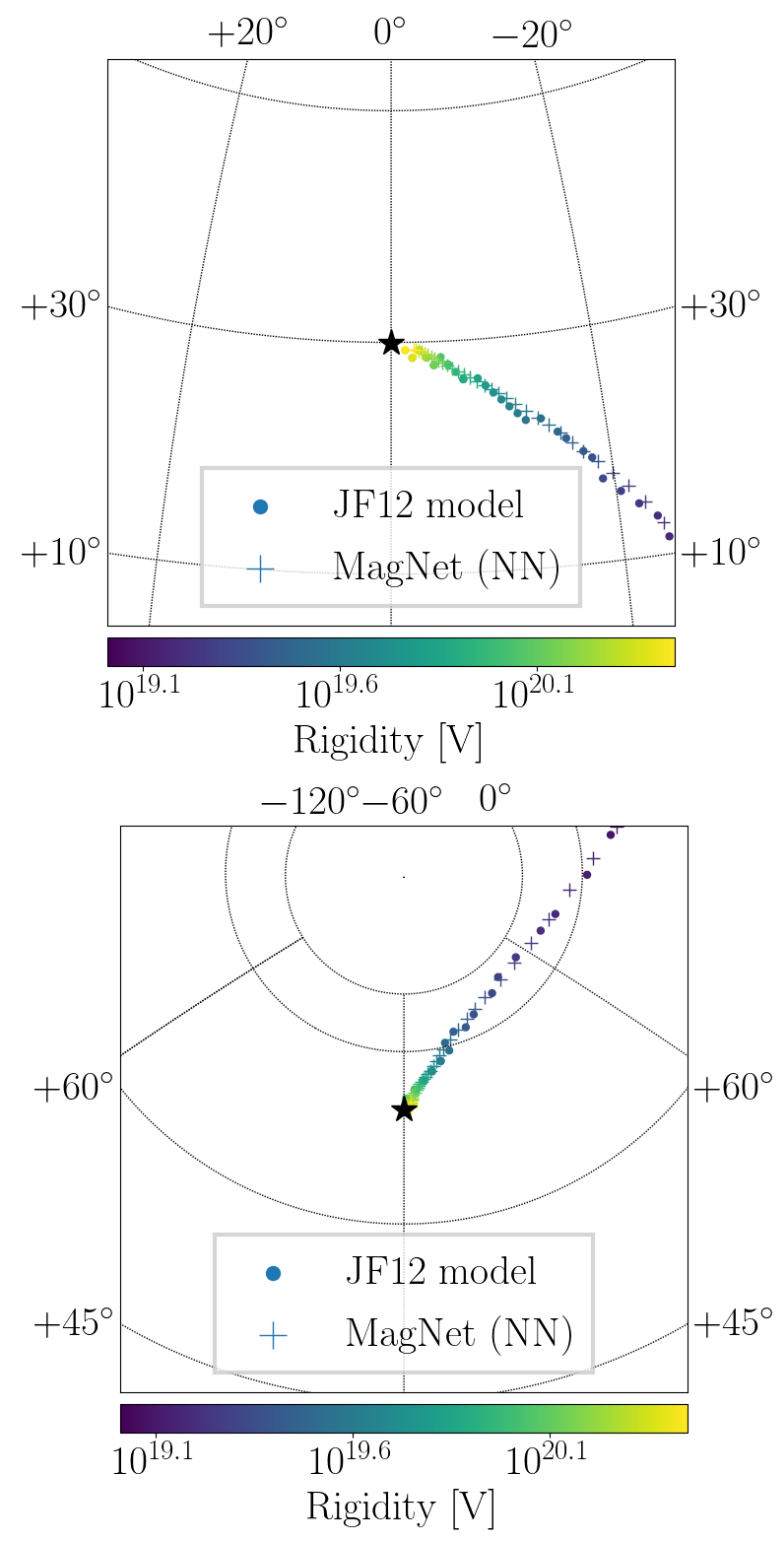

Figure 3. Arrival directions $p_{i}$ for cosmic rays from two arbitrary directions $\hat{s}_{i}$ outside the galaxy denoted by the black star. The circles represent the simulations through the analytical JF12 model with the cosmic ray propagation software CRPropa3 [7], and the cross symbols the predictions of the neural network for different rigidities $R_{i}=\hat{Z}_{i} / E_{i}$.

maximum $X_{\max , i}$ of each cosmic ray, that are however subjected to large shower to shower fluctuations, following an asymmetric distribution, which can be described by Gumbel functions $G\left(A_{i}, E_{i}\right)$ [8]. As event by event fluctuations are large, we can only constrain the cosmic rays to lay consistent within their individual Gumbel distribution. Thus, we formulate an objective term, that penalizes the deviation from $\chi^{2} / N=1$ :

$$
Q=\left[\frac{1}{N} \sum_{i} \frac{\left(X_{\max , i}-\mu_{i}\right)^{2}}{\operatorname{Var}\left(G\left(\hat{A}_{i}, E_{i}\right)\right)}-1\right]^{2}
$$

In this context $\mu_{i}=\arg \max _{X_{\max }} G\left(\hat{A}_{i}, E_{i}\right)$ denotes the most probable $X_{\max }$ value and $\operatorname{Var}\left(G\left(\hat{A}_{i}, E_{i}\right)\right)$ the Gaussian approximation of the variance for either the left or the right tail of the Gumbel distribution. To relate the fitted nuclear charges to mass numbers, we use $A_{i}=2 \cdot Z_{i}$ for $Z_{i} \geq 2$ and to cover the proton case we use $A_{i}=Z_{i}^{2}$ for charges $Z_{i}<2$. Both parts are smoothly connected by sigmoid transition functions.

Clustering: spatial distances to common original directions. While the previous two objective terms are designed to be compatible with the measurements, the cluster term forces cosmic rays to originate from common sources:

$$
C=\frac{\sum_{i} \sum_{j} \epsilon_{i j} \cdot\left\|\hat{s}_{i}-\hat{s}_{j}\right\|^{2}}{\sum_{i} \sum_{j} \epsilon_{i j}}
$$

Here $\epsilon_{i j}$ serves as a weight between cosmic ray $i$ and cosmic ray $j$ which decreases with their spatial distance and depends on their relative constellation to the direction of the deflection in the local magnetic field. One can think about $\epsilon_{i j}$ as an elliptical shaped gaussian with width of $40^{\circ}$ in the direction of the magnetic field deflection and $4^{\circ}$ in orthogonal direction.

Total objective function of the fit. The fit is driven by an objective function consisting of the objective terms (2), (3) and (4). Their relative weights are given by the hyperparameters $\lambda_{Q}$ and $\lambda_{C}$, which should be adjusted in their magnitude to the individual application:

$$
J=D+\lambda_{Q} Q+\lambda_{C} C
$$

For the following application the parameters were set to use $\lambda_{Q}=0.1$ and $\lambda_{C}=0.01$.

\section{Application to a simulated astrophysical scenario}

We apply the fit method to a simulated scenario of 4 sources each emitting 25 cosmic rays overlayed with 900 cosmic rays following an isotropic distribution. The energy spectrum follows a parametrized broken power-law fitted to the data of the Pierre Auger Observatory [9], and applying a lower energy cut of $40 \mathrm{EeV}$. We use an energy-independent mixed composition between proton and oxygen $(Z=1, \ldots, 8)$ that follows a uniform charge distribution. Also here we use the Gumbel distributions $G\left(A_{i}, E_{i}\right)$ to assign an individual shower depth maximum value $X_{\max , i}$ to the cosmic rays.

Cosmic ray deflections in the galactic magnetic field are performed in the JF12 regular field [4], using rigiditydependent magnetic lenses [10]. Additionaly we apply a gaussian rigidity dependent smearing of $\sigma=0.5$. $Z / E[\mathrm{EeV}]$ rad on the arrival directions to account for turbulent intergalactic and galactic magnetic fields. A simulated example arrival map in galactic coordinates is shown in Fig. $4 a$, where the stars denote the 4 source directions, the color code the original charge, and the energy is encoded in the size of the events. Cosmic rays coming from one of the sources are indicated by gray shaded areas.

For the fit, we initialize the charges by applying Bayes' theorem to the shower depth maximum $X_{\max , i}$, assuming a 

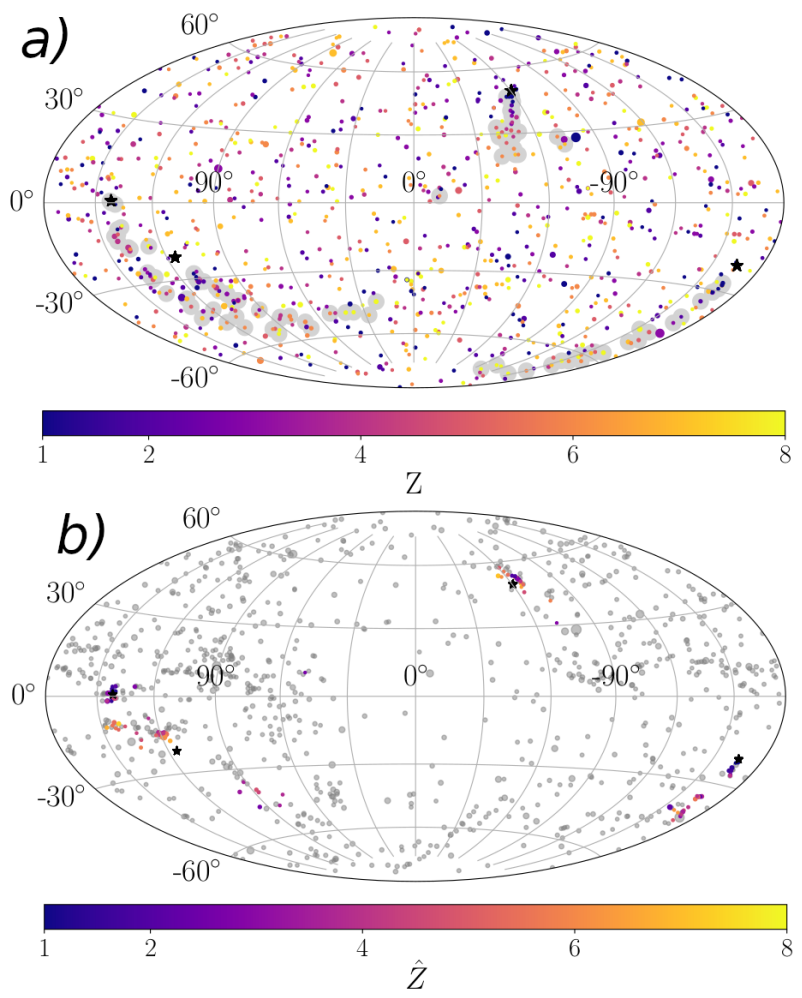

Figure 4. Skymaps in galactic coordinates showing a) the simulated arrival scenario with signal cosmic rays denoted by gray background and b) fitted source directions $\hat{s}_{i}$ with colored symbols referring to the signal events. The star symbols denote the source directions, the color code the charge of the cosmic rays, and the size is proportional to the energy of the cosmic ray.

flat composition prior between $\hat{Z}_{i}=1 \ldots 5$. The initial source directions are simply set to the respective cosmic ray arrival direction, thus $\hat{s}_{i}=p_{i}$.

After approximate $10^{6}$ iterations, the fit converges to values of $\left(\hat{s}_{i}, \hat{Z}_{i}\right)$ as visualized in Fig. $4 \mathrm{~b}$. One can see that the signal cosmic rays largely cluster in regions close to their original source. However, also the isotropic cosmic rays form clusters as demanded by equation (4).
We compare the cluster strength arising in 100 signal scenarios to the ones arising after performing the fit to 500 scenarios of isotropic arrival driections. The cluster strength is here defined in form of a tophat counting with a $5^{\circ}$ tophat-circle around each cosmic ray. For each scenario we then count the number of cosmic rays that exceed a 5 times higher cluster density as the average isotropic cluster density at the same direction in the sky. We find that only 1 out of the 500 isotropic scenarios exceeds the median value that we obtain from the 100 signal scenarios.

\section{Conclusion}

We present a new combined fit method to contract origins of ultra-high energy cosmic rays by varying their charge while keeping them consistent with shower depth maximum measurements. In a simulated astrophysical scenario with a faint signal contribution from a few sources in the sky, we show that the method is capable to successfully cluster the signal cosmic rays at their true source direction.

\section{References}

[1] A. Aab et al. (The Pierre Auger Collaboration), 2017, Science 357 no.6537, 1266

[2] A. Aab et al. (The Pierre Auger Collaboration), 2018, Astrophys. J. 853, L29

[3] M. S. Pshirkov, P. G. Tinyakov, P. P. Kronberg, K. J. Newton-McGee, 2011, Astrophys. J., 738, 192

[4] R. Jansson, G. R. Farrar, 2012, Astrophys. J., 757, 14

[5] K. Ferriere, P. Terral, 2017, A\&A 600, A29

[6] P. Abreu et al. (The Pierre Auger Collaboration), 2012, Astropart. Phys. 35 354-361

[7] R. A. Batista, A. Dundovic, M. Erdmann, et al., 2016, JCAP 05, 38

[8] M. De Domenico, M. Settimo, S. Riggi, E. Bertin, 2013, JCAP 1307) 050

[9] J. Abraham, P. Abreu, M. Aglietta, et al. (The Pierre Auger Collaboration), 2010, Phys. Lett. B 685, 239

[10] H.-P. Bretz, M. Erdmann, P. Schiffer, D. Walz, T. Winchen, 2014, Astropart. Phys. 54, 110 